\title{
Gold-catalyzed oxidation of arylallenes: Synthesis of quinoxalines and benzimidazoles
}

\author{
Dong-Mei Cui ${ }^{* 1}$, Dan-Wen Zhuang ${ }^{1}$, Ying Chen ${ }^{1}$ and Chen Zhang ${ }^{2}$
}

\section{Full Research Paper}

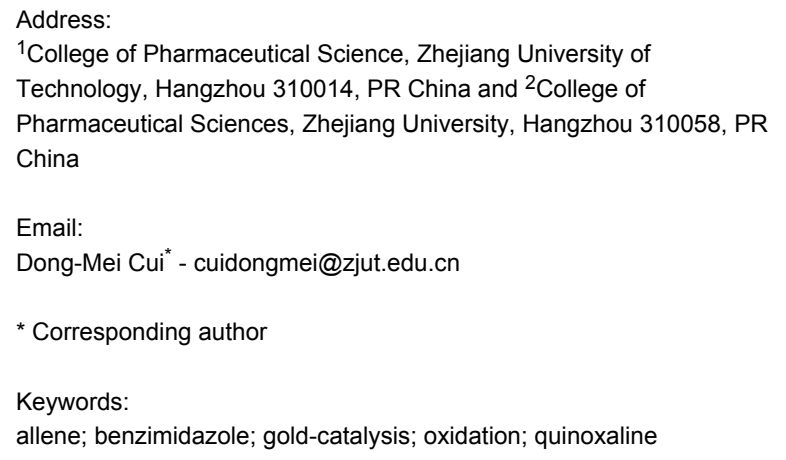

${ }^{1}$ College of Pharmaceutical Science, Zhejiang University of Technology, Hangzhou 310014, PR China and ${ }^{2}$ College of Pharmaceutical Sciences, Zhejiang University, Hangzhou 310058, PR China

Email:

Dong-Mei Cui ${ }^{*}$ - cuidongmei@zjut.edu.cn

* Corresponding author

Keywords:

allene; benzimidazole; gold-catalysis; oxidation; quinoxaline

\author{
Open Access \\ Beilstein J. Org. Chem. 2011, 7, 860-865. \\ doi:10.3762/bjoc. 7.98 \\ Received: 30 March 2011 \\ Accepted: 03 June 2011 \\ Published: 24 June 2011 \\ This article is part of the Thematic Series "Gold catalysis for organic \\ synthesis". \\ Guest Editor: F. D. Toste \\ (C) 2011 Cui et al; licensee Beilstein-Institut. \\ License and terms: see end of document.
}

\begin{abstract}
A gold-catalyzed oxidation of arylallenes to form $\alpha$-diketones and aldehydes in good yields is presented. Further directed synthesis of quinoxalines and benzimidazoles, via the condensation of the resulting $\alpha$-diketones and aldehydes with benzene-1,2-diamine, was achieved in high yields.
\end{abstract}

\section{Introduction}

Recently, several research groups have developed goldcatalyzed homogeneous catalytic reactions [1]. A variety of organic transformations have been shown to be mediated by gold(I) or gold(III) complexes in solution. In addition to its ability to activate unsaturated $\mathrm{C}-\mathrm{C}$ bonds, the catalysis of nucleophilic addition by gold complexes for the formation of carbon-carbon and carbon-heteroatom bonds has been one of the most investigated reactions in recent organometallic catalysis [1-24]. In particular, water as a nucleophilic reagent has been used in the addition of alkynes and allenes [16-18]. In contrast, gold-catalyzed oxidation chemistry has been less well developed [25-36], although oxidative cleavage of carbon-carbon double bonds and carbon-carbon triple bonds by homogeneous gold catalysts was reported recently [28,29,33]. To the best of our knowledge, gold and other transition metalcatalyzed oxidations of allenes have not been reported [37,38]. In the context of ongoing studies on metal-catalyzed atomeconomical reactions, we have been interested in the use of gold for simple and highly efficient transformations. Additionally, quinoxaline and benzimidazole skeletons are common building blocks for the preparation of substances with pronounced biological activities [39-44]. Herein, we report the gold(I)catalyzed oxidation/hydration and oxidative cleavage of allenes to form $\alpha$-diketones and aldehydes, and the synthesis of quinoxalines and benzimidazoles via the condensation of the resulting $\alpha$-diketones and aldehydes with benzene-1,2-diamine [45-56]. 


\section{Results and Discussion}

Our initial explorations focused on the reaction of 4-butylphenylallene (1a) $(0.5 \mathrm{mmol})$ in the presence of a catalytic mixture of $\left(\mathrm{Ph}_{3} \mathrm{P}\right) \mathrm{AuCl}(2 \mathrm{~mol} \%), \mathrm{AgBF}_{4}(8 \mathrm{~mol} \%)$, and $\mathrm{H}_{2} \mathrm{SO}_{4}(0.5 \mathrm{~mol} \%)$ in dioxane $(1.0 \mathrm{~mL})$ and water $(10$ $\mathrm{mmol})$, at $60{ }^{\circ} \mathrm{C}$ for $24 \mathrm{~h}$ in air. This proceeded efficiently to form a 44:56 mixture of $\alpha$-diketone $\mathbf{2 a}$ and aldehyde $\mathbf{3 a}$ in $70 \%$ combined yield (Scheme 1, Table 1, entry 1). The use of either the gold or silver pre-catalyst alone gave lower yields (Table 1 , entries 18 and 19). These results indicate that both the $\mathrm{Au}$ source and $\mathrm{AgBF}_{4}$ play a crucial role in this oxidation. The superior efficiency of the tetrafluoroborate anion was demonstrated by a comparison with other weakly or non-coordinating counter anions. In addition, a change of the counter anion to $\mathrm{OTf}^{-}, \mathrm{SbF}_{6}{ }^{-}$, or $\mathrm{NTf}_{2}^{-}$was also effective (Table 1, entries 2-4). The use of other gold catalysts, e.g., $\left(\mathrm{Ph}_{3} \mathrm{P}\right) \mathrm{AuNO}_{3}$ and $\mathrm{IMesAuCl}$, led to only to combined yields of $\mathbf{2 a}$ and $\mathbf{3 a}$ of $49 \%$ and $60 \%$, respectively (Table 1 , entries 16-17). Decreasing the amount of the sulfuric acid also resulted in a lower yield, although the addition of a large amount of the acid did not affect the reaction (Table 1, entries 8-9). Different acids were screened (Table 1, entries 1, 5-7) and sulfuric acid was found to be the most effective. The use of solvents such as THF, toluene, DCE or ether resulted in a lower conversion (Table 1, entries 10-13). Treatment of $\mathbf{1 a}$ in an atmosphere of $\mathrm{O}_{2}$ (1 atm) afforded 2a and 3a in a combined yield of $47 \%$ (Table 1, entry $20)$. When the reaction was conducted under a nitrogen atmosphere, only trace of products were observed (Table 1, entry 21).

In order to assess the scope of this process, we examined the oxidation of several aryallenes under the optimized conditions indicated in entry 1 of Table 1 . The results are summarized in Table 2. Phenylallene gave a good isolated yield of 1-phenylpropan-1,2-dione (2c) and benzaldehyde (3c) in a ratio of 43:57

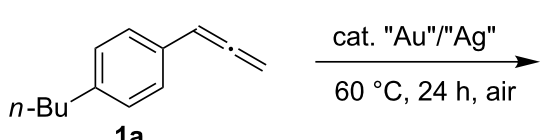

1 a<smiles>CC(=O)C(=O)c1ccc(Br)cc1</smiles>

$2 a$<smiles>O=Cc1ccc(Br)cc1</smiles>

$3 a$

Scheme 1: Oxidation of 4-butylphenylallene.

\begin{tabular}{|c|c|c|c|c|c|c|c|}
\hline Entry & $\begin{array}{l}\mathrm{Au} \\
(2 \mathrm{~mol} \%)\end{array}$ & $\begin{array}{l}\mathrm{Ag} \\
(8 \mathrm{~mol} \%)\end{array}$ & $\begin{array}{l}\mathrm{H}_{2} \mathrm{O} \\
\text { (equiv) }\end{array}$ & $\begin{array}{l}\text { Acid } \\
(\mathrm{mol} \%)\end{array}$ & Solvent & $\begin{array}{l}\text { Ratiob }^{b} \\
2 \mathbf{a}: 3 \mathbf{a}\end{array}$ & $\begin{array}{l}\text { Yield }(\%)^{c} \text { of } \\
2 a \text { and } 3 a\end{array}$ \\
\hline 1 & $\left(\mathrm{PPh}_{3}\right) \mathrm{AuCl}$ & $\mathrm{AgBF}_{4}$ & 20 & $\mathrm{H}_{2} \mathrm{SO}_{4}(0.5)$ & dioxane & $44: 56$ & 70 \\
\hline 2 & $\left(\mathrm{PPh}_{3}\right) \mathrm{AuCl}$ & AgOTf & 20 & $\mathrm{H}_{2} \mathrm{SO}_{4}(0.5)$ & dioxane & $38: 62$ & 63 \\
\hline 3 & $\left(\mathrm{PPh}_{3}\right) \mathrm{AuCl}$ & $\mathrm{AgNTf}_{2}$ & 20 & $\mathrm{H}_{2} \mathrm{SO}_{4}(0.5)$ & dioxane & $55: 45$ & 43 \\
\hline 4 & $\left(\mathrm{PPh}_{3}\right) \mathrm{AuCl}$ & $\mathrm{AgSbF}_{6}$ & 20 & $\mathrm{H}_{2} \mathrm{SO}_{4}(0.5)$ & dioxane & $49: 51$ & 47 \\
\hline 5 & $\left(\mathrm{PPh}_{3}\right) \mathrm{AuCl}$ & $\mathrm{AgBF}_{4}$ & 20 & $\mathrm{~F}_{3} \mathrm{CCO}_{2} \mathrm{H}(0.5)$ & dioxane & $43: 57$ & 58 \\
\hline 6 & $\left(\mathrm{PPh}_{3}\right) \mathrm{AuCl}$ & $\mathrm{AgBF}_{4}$ & 20 & $\mathrm{MsOH}(0.5)$ & dioxane & $39: 61$ & 68 \\
\hline 7 & $\left(\mathrm{PPh}_{3}\right) \mathrm{AuCl}$ & $\mathrm{AgBF}_{4}$ & 20 & $\mathrm{TsOH}(0.5)$ & dioxane & $37: 63$ & 48 \\
\hline 8 & $\left(\mathrm{PPh}_{3}\right) \mathrm{AuCl}$ & $\mathrm{AgBF}_{4}$ & 20 & $\mathrm{H}_{2} \mathrm{SO}_{4}(0.25)$ & dioxane & $49: 51$ & 40 \\
\hline 9 & $\left(\mathrm{PPh}_{3}\right) \mathrm{AuCl}$ & $\mathrm{AgBF}_{4}$ & 20 & $\mathrm{H}_{2} \mathrm{SO}_{4}(1.0)$ & dioxane & $48: 52$ & 70 \\
\hline 10 & $\left(\mathrm{PPh}_{3}\right) \mathrm{AuCl}$ & $\mathrm{AgBF}_{4}$ & 20 & $\mathrm{H}_{2} \mathrm{SO}_{4}(0.5)$ & THF & $47: 53$ & 19 \\
\hline 11 & $\left(\mathrm{PPh}_{3}\right) \mathrm{AuCl}$ & $\mathrm{AgBF}_{4}$ & 20 & $\mathrm{H}_{2} \mathrm{SO}_{4}(0.5)$ & toluene & $39: 61$ & 49 \\
\hline 12 & $\left(\mathrm{PPh}_{3}\right) \mathrm{AuCl}$ & $\mathrm{AgBF}_{4}$ & 20 & $\mathrm{H}_{2} \mathrm{SO}_{4}(0.5)$ & DCE & $43: 57$ & 60 \\
\hline 13 & $\left(\mathrm{PPh}_{3}\right) \mathrm{AuCl}$ & $\mathrm{AgBF}_{4}$ & 20 & $\mathrm{H}_{2} \mathrm{SO}_{4}(0.5)$ & ether & $36: 64$ & 37 \\
\hline 14 & $\left(\mathrm{PPh}_{3}\right) \mathrm{AuCl}$ & $\mathrm{AgBF}_{4}$ & 10 & $\mathrm{H}_{2} \mathrm{SO}_{4}(0.5)$ & dioxane & $46: 54$ & 32 \\
\hline 15 & $\left(\mathrm{PPh}_{3}\right) \mathrm{AuCl}$ & $\mathrm{AgBF}_{4}$ & 40 & $\mathrm{H}_{2} \mathrm{SO}_{4}(0.5)$ & dioxane & $53: 47$ & 39 \\
\hline 16 & $\left(\mathrm{PPh}_{3}\right) \mathrm{AuNO}_{3}$ & - & 20 & $\mathrm{H}_{2} \mathrm{SO}_{4}(0.5)$ & dioxane & 49:51 & 49 \\
\hline 17 & IMeSAuCI & $\mathrm{AgBF}_{4}$ & 20 & $\mathrm{H}_{2} \mathrm{SO}_{4}(0.5)$ & dioxane & $54: 46$ & 60 \\
\hline 18 & $\mathrm{PPh}_{3} \mathrm{AuCl}$ & - & 20 & $\mathrm{H}_{2} \mathrm{SO}_{4}(0.5)$ & dioxane & $38: 62$ & 15 \\
\hline 19 & - & $\mathrm{AgBF}_{4}$ & 20 & $\mathrm{H}_{2} \mathrm{SO}_{4}(0.5)$ & dioxane & $47: 53$ & 28 \\
\hline $20^{d}$ & $\left(\mathrm{PPh}_{3}\right) \mathrm{AuCl}$ & $\mathrm{AgBF}_{4}$ & 20 & $\mathrm{H}_{2} \mathrm{SO}_{4}(0.5)$ & dioxane & $50: 50$ & 47 \\
\hline $21^{e}$ & $\left(\mathrm{PPh}_{3}\right) \mathrm{AuCl}$ & $\mathrm{AgBF}_{4}$ & 20 & $\mathrm{H}_{2} \mathrm{SO}_{4}(0.5)$ & dioxane & - & trace \\
\hline
\end{tabular}

aAll reactions were carried out using $1 \mathrm{a}(0.5 \mathrm{mmol}),\left(\mathrm{Ph}_{3} \mathrm{P}\right) \mathrm{AuCl}(2 \mathrm{~mol} \%), \mathrm{AgBF}_{4}(8 \mathrm{~mol} \%)$, and acid $(0.25-1.0 \mathrm{~mol} \%)$ in solvent $(1.0 \mathrm{~mL})$ and water $(0.5-1.0 \mathrm{mmol})$ at $60^{\circ} \mathrm{C}$ for $24 \mathrm{~h}$. ${ }^{\mathrm{b}}$ The ratio of $\mathbf{2 a}$ and $\mathbf{3 a}$ was determined by GC. Clsolated and combined yield of $\mathbf{2 a}$ and $\mathbf{3 a}$. ${ }^{\mathrm{d}}$ Under an atmosphere of $\mathrm{O}_{2}(1 \mathrm{~atm})$. ${ }^{\mathrm{e}}$ Under an atmosphere of $\mathrm{N}_{2}$. 
Table 2: Oxidation of 1 catalyzed by a mixture of $\left(\mathrm{PPh}_{3}\right) \mathrm{AuCl}, \mathrm{AgBF}_{4}$, and $\mathrm{H}_{2} \mathrm{SO}_{4}$.

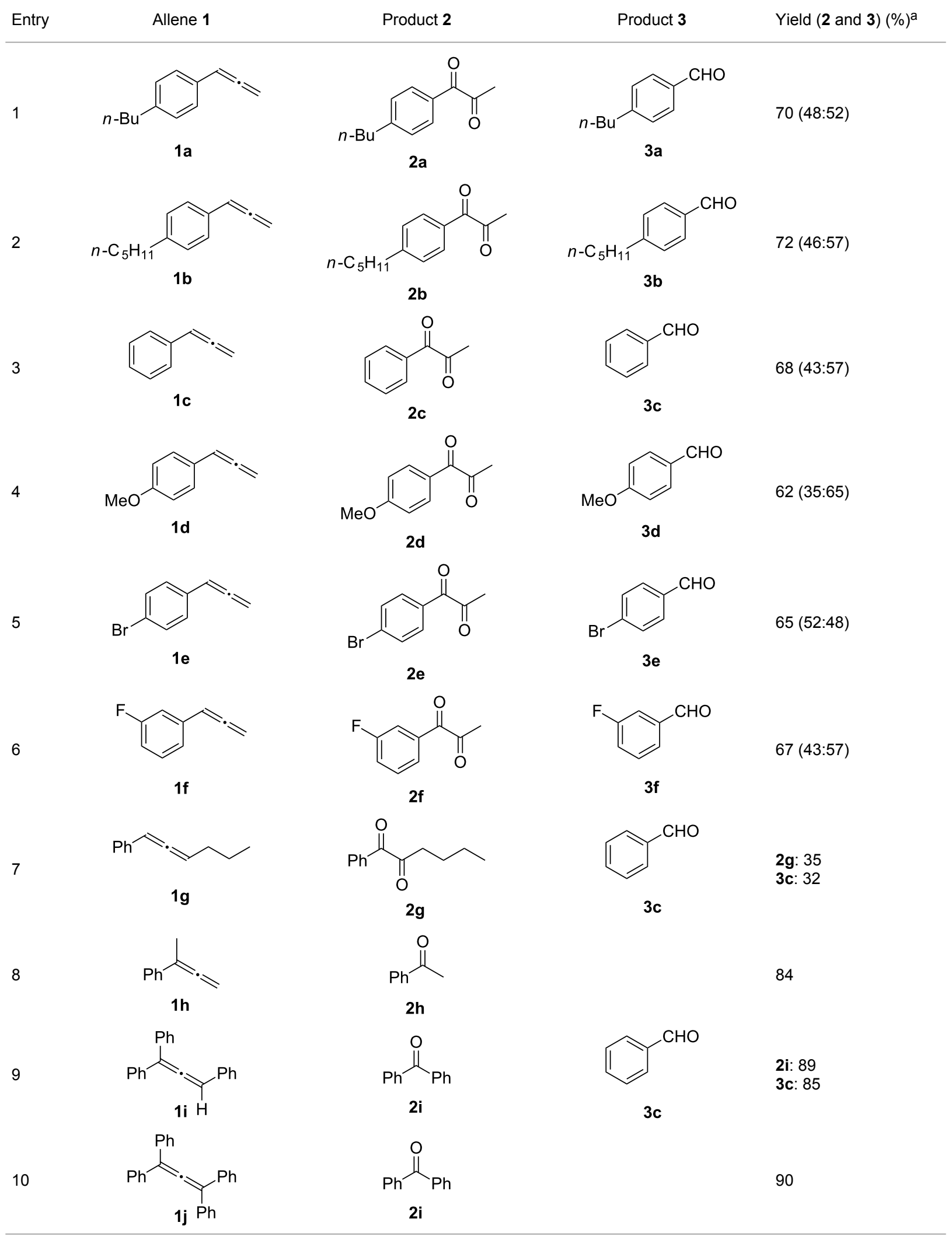

alsolated yield. The ratio of $\mathbf{2}$ and $\mathbf{3}$ in the parentheses was determined by GC. 
(Table 2, entry 3 ). With a more electron-donating alkoxy group, the expected products were again obtained in good yields (Table 2, entry 4). In addition, oxidation of arylallene with an electron-withdrawing fluoro or bromo substituent on the benzene ring also took place smoothly (Table 2, entries 5 and 6). Disubstituted allenes were also examined. Thus, the 1,3-disubstituted allene $\mathbf{1 g}$, was oxidized to afford $\alpha$-diketone $\mathbf{2 g}$ and aldehyde $\mathbf{3 c}$ in $35 \%$ and $32 \%$ yields, respectively (Table 2 , entry 7 ). Similarly, oxidation cleavage of 1,1-disubstituted, trisubstituted and tetrasubstituted allenes gave the expected products (Table 2, entries 8-10). In striking contrast to aromatic allenes, aliphatic allenes, such as hepta-1,2-diene and 1-(propa-1,2dienyl)cyclohex-1-ene failed to undergo Au-catalyzed oxidative transformation under the same reaction conditions.

Having prepared a variety of $\alpha$-diketones and aldehydes successfully, we then undertook the synthesis of quinoxalines and benzimidazoles (Scheme 2). Thus, the treatment of the corresponding mixture of $\alpha$-diketone 2 and aldehyde 3 with benzene-1,2-diamine in the presence of $20 \mathrm{~mol} \%$ oxalic acid afforded the desired quinoxalines 4 and benzimidazoles 5 in high yields (Table 3, entries 1-6).

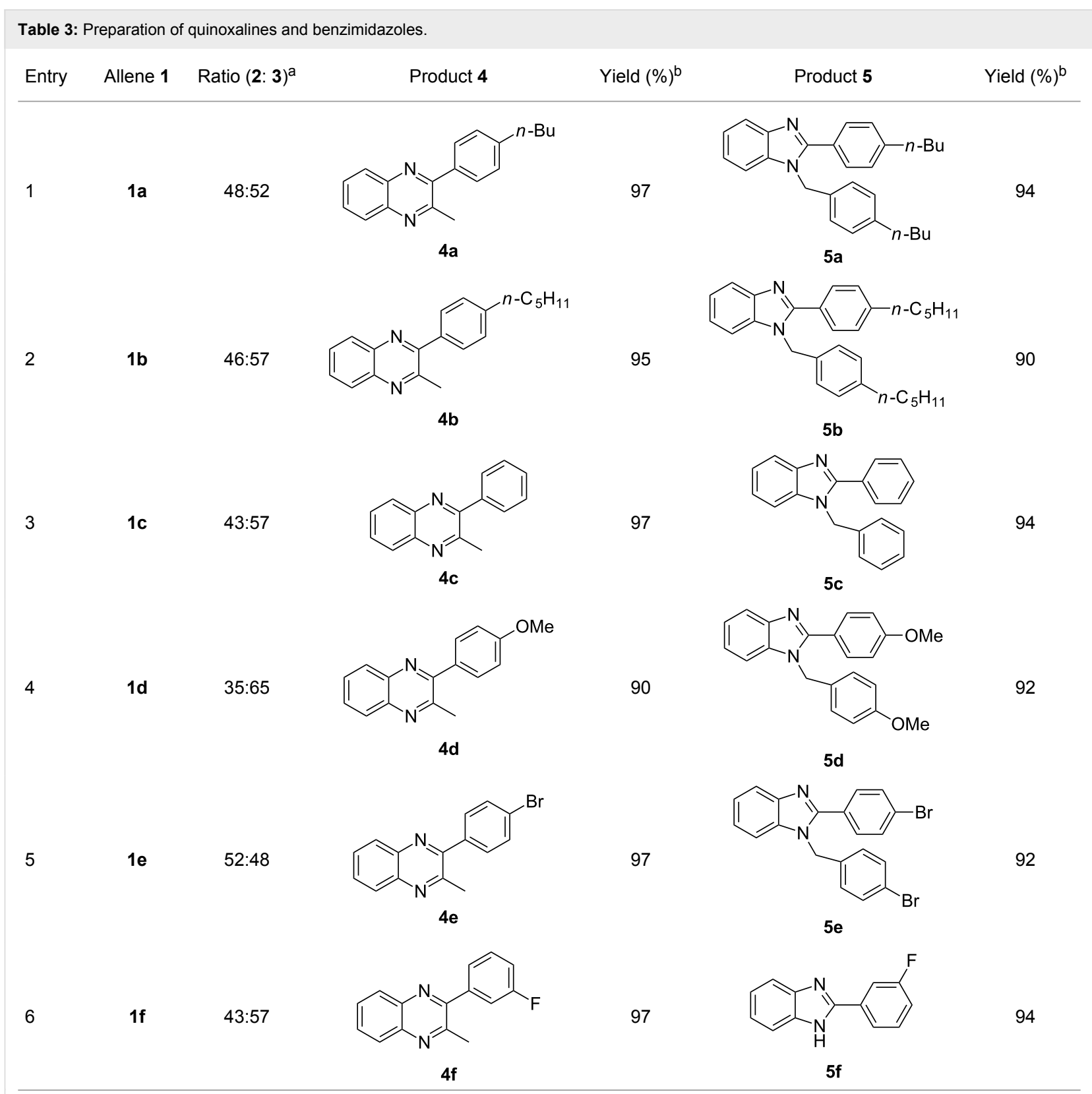

aThe ratio of $\mathbf{2}$ and $\mathbf{3}$ was determined by GC. ${ }^{\text {blsolated yield. }}$ 


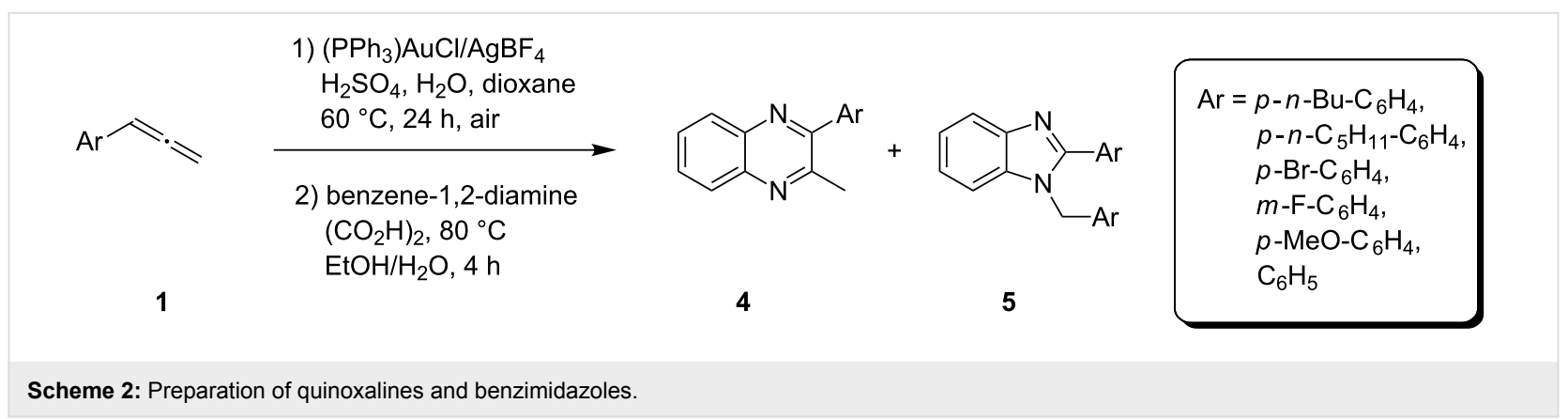

\section{Conclusion}

We have developed a new gold-catalyzed oxidation of arylallenes to give $\alpha$-diketones and aldehydes in good yields. In addition, the directed synthesis of quinoxalines and benzimidazoles via the condensation of the resulting $\alpha$-diketones and aldehydes with benzene-1,2-diamine was achieved in high yields. This reaction appears to proceed via oxidation/hydration and oxidative cleavage of the allene, and investigations into the mechanism of this reaction are underway in our laboratory.

\section{Experimental}

General methods: Unless otherwise noted, materials were obtained from commercial suppliers and used without further purification. Thin layer chromatography (TLC) was performed on silica gel $60 \mathrm{~F}_{254}$ and visualized with UV light. Column chromatography was performed with silica gel (mesh 300-400). ${ }^{1} \mathrm{H}$ NMR and ${ }^{13} \mathrm{C}$ NMR spectra were recorded on a Bruker Avance $500 \mathrm{MHz}$ spectrometer in $\mathrm{CDCl}_{3}$ with $\mathrm{Me}_{4} \mathrm{Si}$ as internal standard. Data are reported as follows: Chemical shift in ppm ( $\delta$ ), multiplicity ( $\mathrm{s}=$ singlet, $\mathrm{d}=$ doublet, $\mathrm{t}=$ triplet, $\mathrm{q}=$ quartet, $\mathrm{br}=$ broad and $\mathrm{m}=$ multiplet $)$, coupling constant in Hertz $(\mathrm{Hz})$ and signal integration. Infrared spectra (IR) were obtained on 370 FT-IR spectrometer; absorptions are reported in $\mathrm{cm}^{-1}$. Mass spectra were obtained under electron impact mode (EI) and high resolution mass spectra were measured on a high resolution mass spectrometer (GCT Premier).

\section{General procedure}

Step A (a typical procedure): Sulfuric acid (0.5 mol \%) was added to a mixture of 4-butylphenylallene $(0.5 \mathrm{mmol})$, water (10 mmol), $\left(\mathrm{PPh}_{3}\right) \mathrm{AuCl}(2 \mathrm{~mol} \%), \mathrm{AgBF}_{4}(8 \mathrm{~mol} \%)$, and dioxane $(1 \mathrm{~mL})$. The mixture was stirred at $60{ }^{\circ} \mathrm{C}$ for 24 , the reaction quenched with a saturated solution of $\mathrm{NaHCO}_{3}$ and extracted with ethyl acetate $(3 \times 10 \mathrm{~mL})$. The combined organic layers were washed with brine, dried over $\mathrm{Na}_{2} \mathrm{SO}_{4}$ and concentrated in vacuo. The residue was purified by flash chromatography to give the desired products $\mathbf{2 a}$ and 3a $(48: 52,63.75 \mathrm{mg}$, $70 \%)$.
Step B (a typical procedure): A mixture of $\mathbf{2 a}$ and 3a (63.75 $\mathrm{mg}$ ), benzene-1,2-diamine (28 $\mathrm{mg}, 0.259 \mathrm{mmol})$, oxalic acid (6.3 $\mathrm{mg}, 0.07 \mathrm{mmol}, 20 \mathrm{~mol} \%$ ), water $(1 \mathrm{~mL})$ was dissolved in ethanol $(1 \mathrm{~mL})$. The mixture was heated under reflux for $4 \mathrm{~h}$. The reaction was quenched with a saturated solution of $\mathrm{NaHCO}_{3}$ and then extracted with ethyl acetate $(3 \times 10 \mathrm{~mL})$. The combined organic layers were washed with brine, dried over $\mathrm{Na}_{2} \mathrm{SO}_{4}$ and concentrated in vacuo. The residue was purified by flash chromatography to give the desired products $4 \mathbf{a}(45.0 \mathrm{mg}$, 97\%) and 5a (33.9 mg, 94\%).

\section{Supporting Information}

\section{Supporting Information File 1}

Analytical and spectroscopic data for new compounds.

[http://www.beilstein-journals.org/bjoc/content/

supplementary/1860-5397-7-98-S1.pdf]

\section{Acknowledgements}

We thank the Natural Science Foundation of China (Nos. 20672099 and 20572094) and Zhejiang Science Foundation (No. Y4100558) for financial support.

\section{References}

1. Gorin, D. J.; Sherry, B. D.; Toste, F. D. Chem. Rev. 2008, 108, 3351. doi:10.1021/cr068430g

2. Jiménez-Núñez, E.; Echavarren, A. M. Chem. Rev. 2008, 108, 3326. doi:10.1021/cr0684319

3. Arcadi, A. Chem. Rev. 2008, 108, 3266. doi:10.1021/cr068435d

4. Li, Z.; Brouwer, C.; He, C. Chem. Rev. 2008, 108, 3239. doi:10.1021/cr068434l

5. Skouta, R.; Li, C.-J. Tetrahedron 2008, 64, 4917. doi:10.1016/j.tet.2008.03.083

6. Shen, H. C. Tetrahedron 2008, 64, 3885. doi:10.1016/j.tet.2008.01.081

7. Muzart, J. Tetrahedron 2008, 64, 5815. doi:10.1016/j.tet.2008.04.018

8. Hashmi, A. S. K. Chem. Rev. 2007, 107, 3180. doi:10.1021/cr000436x

9. Hashmi, A. S. K. Gold Bull. 2004, 37, 51. doi:10.1007/BF03215517

10. Arcadi, A.; Giuseppe, S. D. Curr. Org. Chem. 2004, 8, 795. doi:10.2174/1385272043370564

11. Hashmi, A. S. K.; Hutchings, G. J. Angew. Chem., Int. Ed. 2006, 45, 7896. doi:10.1002/anie.200602454 
12. Shapiro, N. D.; Toste, F. D. J. Am. Chem. Soc. 2007, 129, 4160. doi:10.1021/ja070789e

13. Dubé, P.; Toste, F. D. J. Am. Chem. Soc. 2006, 128, 12062. doi:10.1021/ja064209+

14. Marion, N.; Díez-González, S.; de Frémont, P.; Noble, A. R.; Nolan, S. P. Angew. Chem., Int. Ed. 2006, 45, 3647. doi:10.1002/anie.200600571

15. Zhang, Y.; Donahue, J. P.; Li, C.-J. Org. Lett. 2007, 9, 627. doi:10.1021/ol062918m

16. Mizushima, E.; Sato, K.; Hayashi, T.; Tanaka, M. Angew. Chem., Int. Ed. 2002, 41, 4563. doi:10.1002/1521-3773(20021202)41:23<4563::AID-ANIE4563>3.0.CO ;2-U

17. Casado, R.; Contel, M.; Laguna, M.; Romero, P.; Sanz, S. J. Am. Chem. Soc. 2003, 125, 11925. doi:10.1021/ja036049x

18. Zhang, Z.-B.; Lee, S. D.; Fisher, A. S.; Widenhoefer, R. A. Tetrahedron 2009, 65, 1794. doi:10.1016/j.tet.2008.10.113

19. Liu, B.; De Brabander, J. K. Org. Lett. 2006, 8, 4907. doi:10.1021/ol0619819

20. Mizushima, E.; Hayashi, T.; Tanaka, M. Org. Lett. 2003, 5, 3349. doi:10.1021/ol0353159

21. Arcadi, A.; Di Giuseppe, S.; Marinelli, F.; Rossi, E. Adv. Synth. Catal. 2001, 343, 443. doi:10.1002/1615-4169(200107)343:5<443::AID-ADSC443>3.0.CO;2-\#

22. Hashmi, A. S. K.; Weyrauch, J. P.; Frey, W.; Bats, J. W. Org. Lett. 2004, 6, 4391. doi:10.1021/ol0480067

23. Luo, Y. M.; Li, Z.; Li, C. J. Org. Lett. 2005, 7, 2675. doi:10.1021/ol050826b

24. Lavallo, V.; Frey, G. D.; Donnadieu, B.; Soleilhavoup, M.; Bertrand, G. Angew. Chem., Int. Ed. 2008, 47, 5224. doi:10.1002/anie.200801136

25. Deng, X.-Y.; Friend, C. M. J. Am. Chem. Soc. 2005, 127, 17178. doi:10.1021/ja0557031

26. Guzman, J.; Carrettin, S.; Corma, A. J. Am. Chem. Soc. 2005, 127, 3286. doi:10.1021/ja043752s

27. Guan, B.; Xing, D.; Cai, G.; Wan, X.; Yu, N.; Fang, Z.; Yang, L.; Shi, Z. J. Am. Chem. Soc. 2005, 127, 18004. doi:10.1021/ja055398j

28. Xing, D.; Guan, B.; Cai, G.; Fang, Z.; Yang, L.; Shi, Z. Org. Lett. 2006, 8, 693. doi:10.1021/ol052830t

29. Liu, Y.; Song, F.; Guo, S. J. Am. Chem. Soc. 2006, 128, 11332. doi:10.1021/ja062610q

30. Taduri, B. P.; Sohel, S. M. A.; Cheng, H.-M.; Lin, G.-Y.; Liu, R.-S. Chem. Commun. 2007, 2530. doi:10.1039/b700659d

31. Witham, C. A.; Mauleon, P.; Shapiro, N. D.; Sherry, B. D.; Toste, F. D. J. Am. Chem. Soc. 2007, 129, 5838. doi:10.1021/ja071231+

32. Murakami, Y.; Konishi, K. J. Am. Chem. Soc. 2007, 129, 14401. doi:10.1021/ja075051b

33. Das, A.; Chaudhuri, R.; Liu, R.-S. Chem. Commun. 2009, 4046. doi:10.1039/b908338c

34. Ye, L.; Cui, L.; Zhang, G.; Zhang, L. J. Am. Chem. Soc. 2010, 132, 3258. doi:10.1021/ja100041e

35. Lu, B.; Li, C.; Zhang, L. J. Am. Chem. Soc. 2010, 132, 14070. doi:10.1021/ja1072614

36. Liu, Y.; Song, F.; Song, Z.; Liu, M.; Yan, B. Org. Lett. 2005, 7, 5409. doi:10.1021/ol052160r

37. Erden, I.; Martinez, T. R. Tetrahedron Lett. 1991, 32, 1859 doi:10.1016/S0040-4039(00)85981-X

38. Fleming, S. A.; Liu, R.; Redd, J. T. Tetrahedron Lett. 2005, 46, 8095. doi:10.1016/j.tetlet.2005.09.138

39. Seitz, L. E.; Suling, W. J.; Reynolds, R. C. J. Med. Chem. 2002, 45, 5604. doi:10.1021/jm020310n
40. Hazeldine, S. T.; Polin, L.; Kushner, J.; Paluch, J.; White, K.; Edelstein, M.; Palomino, E.; Corbett, T. H.; Horwitz, J. P. J. Med. Chem. 2001, 44, 1758. doi:10.1021/jm0005149

41. Bailly, C.; Echepare, S.; Gago, F.; Waring, M. J. Anti-cancer Drug Des. 1999, 14, 291.

42. Sato, K.; Shiratori, O.; Katagiri, K. J. Antibiot. 1967, 20, 270.

43. Tamm, I. Science 1957, 126, 1235.

44. Roth, T.; Morningstar, M. L.; Boyer, P. L.; Hughes, S. H.; Buckheit, R. W., Jr.; Michejda, C. J. J. Med. Chem. 1997, 40, 4199 doi:10.1021/jm970096g

45. Dayan, S.; Ben-David, I.; Rozen, S. J. Org. Chem. 2000, 65, 8816. doi:10.1021/jo001101i

46. Wan, Z.; Jones, C. D.; Mitchell, D.; Pu, J. Y.; Zhang, T. Y. J. Org. Chem. 2006, 71, 826. doi:10.1021/j0051793g

47. Yusubov, M. S.; Zholobova, G. A.; Vasilevsky, S. F.; Tretyakov, E. V.; Knight, D. W. Tetrahedron 2002, 58, 1607. doi:10.1016/S0040-4020(02)00025-X

48. Giraud, A.; Provot, O.; Peyrat, J.-F.; Alami, M.; Brion, J.-D. Tetrahedron 2006, 62, 7667. doi:10.1016/j.tet.2006.05.072

49. Mousset, C.; Provot, O.; Hamze, A.; Bignon, J.; Brion, J.-D.; Alami, M. Tetrahedron 2008, 64, 4287. doi:10.1016/j.tet.2008.02.081

50. Niu, M.; Fu, H.; Jiang, Y.; Zhao, Y. Synthesis 2008, 2879. doi:10.1055/s-2008-1067240

51. Tan, K. J.; Wille, U. Chem. Commun. 2008, 6239. doi:10.1039/B815358B

52. Ren, W.; Xia, Y.; Ji, S.-J.; Zhang, Y.; Wan, X.; Zhao, J. Org. Lett. 2009 , 11, 1841. doi:10.1021/ol900344g

53. Al-Rashid, Z. F.; Johnson, W. L.; Hsung, R. P.; Wei, Y.; Yao, P.-Y.; Liu, R.; Zhao, K. J. Org. Chem. 2008, 73, 8780. doi:10.1021/jo8015067

54. Ren, W.; Liu, J.; Chen, L.; Wan, X. Adv. Synth. Catal. 2010, 352, 1424. doi:10.1002/adsc.201000250

55. Mori, S.; Takubo, M.; Yanase, T.; Maegawa, T.; Monguchi, Y.; Sajiki, H. Adv. Synth. Catal. 2010, 352, 1630. doi:10.1002/adsc.201000173

56. Hasaninejad, A.; Zare, A.; Mohammadizadeh, M. R.; Shekouhy, M. ARKIVOC 2008, (xiii), 28.

\section{License and Terms}

This is an Open Access article under the terms of the Creative Commons Attribution License (http://creativecommons.org/licenses/by/2.0), which permits unrestricted use, distribution, and reproduction in any medium, provided the original work is properly cited.

The license is subject to the Beilstein Journal of Organic Chemistry terms and conditions:

(http://www.beilstein-journals.org/bjoc)

The definitive version of this article is the electronic one which can be found at: $\underline{\text { doi: } 10.3762 / \text { bjoc. } 7.98}$ 